\title{
Alveolar Soft Part Sarcomas: Molecular Pathogenesis and Implications for Novel Targeted Therapies
}

\author{
Bryan Mitton $^{1}$ and Noah Federman ${ }^{1,2}$ \\ ${ }^{1}$ Division of Pediatric Hematology/Oncology, Department of Pediatrics, Mattel Children's Hospital at UCLA, \\ UCLA David Geffen School of Medicine, 10833 Le Conte Avenue, Los Angeles, CA 90095-175, USA \\ ${ }^{2}$ UCLA Pediatric Bone and Soft Tissue Sarcoma Program, The UCLA Sarcoma Program, Nanotechnology Program Area, \\ UCLA Jonsson Comprehensive Cancer Center, Los Angeles, CA 90095, USA
}

Correspondence should be addressed to Noah Federman, nfederman@mednet.ucla.edu

Received 2 December 2011; Accepted 29 January 2012

Academic Editor: C. Fisher

Copyright (๑) 2012 B. Mitton and N. Federman. This is an open access article distributed under the Creative Commons Attribution License, which permits unrestricted use, distribution, and reproduction in any medium, provided the original work is properly cited.

\begin{abstract}
Alveolar soft part sarcoma (ASPS) is a very rare soft tissue sarcoma which arises primarily in children and young adults. Despite its unique histology and well-characterized genetic translocation, many questions remain regarding the pathogenesis and treatment of this tumor type. Though collective clinical experience with this tumor type spans more than 60 years, there has been little progress made in treating this uncommon but frequently fatal disease. This paper focuses on the available data regarding its molecular pathogenesis and insights into targeted therapeutics as well as the results of clinical trials performed to date to hopefully improve the outcome of patients with this rare malignancy.
\end{abstract}

\section{Introduction}

Alveolar soft part sarcoma (ASPS) is a very rare sarcoma which arises primarily in children and young adults. Despite more than 60 years of experience with ASPS, several fundamental questions regarding this tumor type remain unanswered. The tissue of origin for ASPS remains unclear; the risk factors which lead to tumorigenesis and clinical progression are unknown, and the optimal approach to therapy is undefined. Though significant progress has been made in the molecular characterization of this tumor in the past 10 years and a number of exciting clinical trials are underway, this tumor has eluded elementary characterization for many decades.

\section{Clinical Features of Alveolar Soft Part Sarcomas}

Alveolar soft part sarcoma (ASPS) accounts for approximately $0.5-1 \%$ of all soft tissue sarcomas [1]. It is diagnosed most commonly in those between 15 and 35 years of age; in some large case series, the incidence is slightly increased in young females by a ratio of $3: 2$ compared to age-matched males [2]. Disease usually presents as a painless, soft, slowgrowing lesion that rarely causes functional impairment. In children, ASPS most frequently occurs in the head and neck region, especially the tongue or orbit; in older adults, it arises from muscles of the lower or upper extremities [3-5].

Typically, this tumor grows indolently for years. Metastasis is detected in $\sim 20 \%$ of patients at diagnosis and develops in $\sim 80 \%$ of patients during the course of treatment [2]. Risk factors for developing this tumor remain undefined, but the risk for metastatic disease includes older age and larger tumor size $(>5 \mathrm{~cm})$ at diagnosis $[2,6]$. ASPS, as with most other sarcomas, most often metastasizes to the lungs, but central nervous system involvement is also frequently described; indeed, ASPS has been reported to metastasize to the brain more frequently than any other form of high-grade sarcoma [1, 7-9]. Though there have been no cases reported of brain metastasis in the absence of lung metastasis, liver metastasis and intraosseous extension of the tumor without widespread disease have been described. The primary tumors are often large, with a mean size of $6.5 \mathrm{~cm}$ in one study, and typically high vascular, such that they sometimes present as a pulsatile mass [2]. On magnetic resonance imaging, they 
may appear similar to arteriovenous malformations [10]. Irregular intravascular extension is present at the tumor margins in almost all cases. The 5-year overall survival rates range from 45 to $88 \%$, with a 20 -year survival of approximately $15 \%$; the median survival time is 6 years. Survival is dictated largely by disease stage and the size of the primary tumor [1$3,6]$.

\section{Histologic Features of Alveolar Soft Part Sarcomas}

Christopherson et al. were the first to designate these tumors as "alveolar soft part sarcomas" in 1952, given their unique histologic appearance and uncertain tissue origin [11]. To date, the definitive origin of this tumor remains unknown. There is some immunohistochemical evidence suggesting that ASPS may arise from striated muscle or pericytes, this remains controversial [12-15]. Primary ASPS tumor sites have also been reported in tissues where skeletal muscle is absent, such as in the stomach, breast tissue, and the female genital tract [16-18].

ASPS tumors are histologically distinctive. Interestingly, this tumor type was originally named for its striking architectural similarity to respiratory alveoli; classically, poorly differentiated tumor cells are arranged in nests separated by thin layers of connective tissue containing sinusoidal vascular channels, which in turn are lined by thin endothelium [3]. A histologic variant of ASPS has been described in young patients with lingual ASPS, which lacks the typical cellular discohesion and thus has a solid "nonalveolar" growth pattern [19]. Smetana and Scott in 1951 were the first to describe the hallmark intracytoplasmic crystals of ASPS [20]. These crystals are rod shaped, coarse, and basophilic bodies of unknown significance, though they have been shown to contain monocarboxylate transporter 1 and CD147 [21]. These cells demonstrate PAS-positive granules in almost all tumors and often stain positively for desmin [4]. Electron microscopy demonstrates rhomboid, rod shaped crystals consisting of rigid fibrils. In spite of these features, ASPS still may present a diagnostic challenge, as it may resemble metastatic renal cell carcinoma, paragangliomas, granular cell tumors, or melanomas [3]. Preoperative imaging, usually with magnetic resonance imaging, is the standard of care. Core needle biopsy or fine needle aspiration should be considered before definitive surgery. Because of the presence of intracellular crystals, fine-needle cytology can often offer sufficient material for diagnosis, but as with any diagnosis of solid tumor, excisional biopsy may be required to diagnose this rare tumor.

We now turn our attention to the available data concerning the pathogenesis of this unique tumor, as well as the therapeutic strategies now available.

\section{Molecular Pathogenesis of Alveolar Soft Part Sarcoma}

ASPS is characterized by an unbalanced translocation between the $\mathrm{X}$ chromosome and chromosome 17, first described in a seminal paper by Ladanyi et al. in 2001 [22]. The $\operatorname{der}(17) \mathrm{t}(\mathrm{X} ; 17)(\mathrm{p} 11 ; 25)$ translocation is found in all ASPS tumors studied; in the majority of ASPS tumors, this translocation is found in an unbalanced form, resulting in loss of heterozygosity at 11q25 [23]. Interestingly, this translocation is also found in a distinctive subset of renal cell carcinomas which frequently have papillary architecture, usually in the balanced form [24].

Elegant studies defined the precise base pair position at which this translocation occurs; the resultant fusion protein involves the Alveolar Soft Part Sarcoma Critical Region-1 gene (ASPSCR-1) located on chromosome 17q25 and the Transcription Factor for Immunoglobulin HeavyChain Enhancer 3 (TFE3) gene, located on chromosome Xp11.22 [22]. Structurally, the N-terminus of the ASPSCR1 gene is fused in-frame with the TFE3 (Transcription Factor E3) gene at exon 3 (Type I) or exon 4 (Type 2), resulting in one of two novel, functional ASPSCR1-TFE3 fusion proteins which are capable of inducing aberrant transcription of TFE3-regulated genes (Figure 1). At the molecular level, the first 234 aminoterminal aminoacids from ASPSCR-1 are fused to the TFE3 gene at aminoacid positions 280 or 315 . There are no data as to whether these two different fusion products result in clinically different diseases in terms of presentation, metastasis, or prognosis.

The TFE3 gene is a member of the microphthalmia transcription factor/transcription factor E (MITF-TFE) family of basic helix-loop-helix leucine zipper (bHLH-Zip) transcription factors along with MITF, TFEB, and TFEC [25]. The TFE3 gene expresses the TEF3 (Transcription Enhancing Factor 3) protein. The MITF-TFE family of transcription factors functions as homo- or heterodimers. They are ubiquitously expressed, and each of the possible binding pairs have been demonstrated in vitro; it has been suggested the specific gene program activated by the TEF3 protein depends mainly on its binding partners, which is dynamic and tissue specific [26]. The TEF3 protein, encoded by the TFE3 gene, also interacts with transcriptional regulators such as E2F3, SMAD3, and LEF-1, and plays a variety of roles in cell growth and proliferation [27].

A number of clues suggest the potential of the TFE3 gene as a protooncogene. Early evidence demonstrated that the TEF3 protein activates transcription via binding of its E3 motif to the EBox DNA consensus sequence (CANNTG) in the immunoglobulin heavy-chain enhancer [28]. TEF3 regulates a number of metabolic genes which possess the EBox in their promoters, such as the S-phase regulator cyclin E, in an E2F3-dependent manner [27]. Interestingly, TEF3 may confer resistance to cell cycle arrest signals and can override arrest when ectopically expressed. For example, the presence of TEF3 can override Rb-induced cell cycle arrest, and can block the antimitogenic effects of TGF- $\beta$ in mammalian cells [27]. TEF3 has an activating domain at both the Nand C-termini; in vitro deletion of the $\mathrm{N}$-terminal domain results in a dominant negative form of the factor that interferes with the function of the full-length protein [29]. This activation domain is lost in the Type 1 ASPSCR1-TFE3 gene translocation and not the Type 2 variant, though there are no clear phenotypic differences in the tumors that arise 

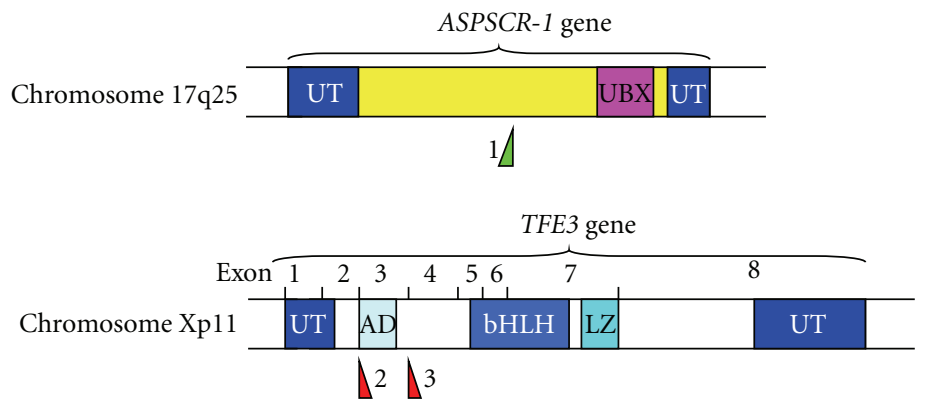

(a)

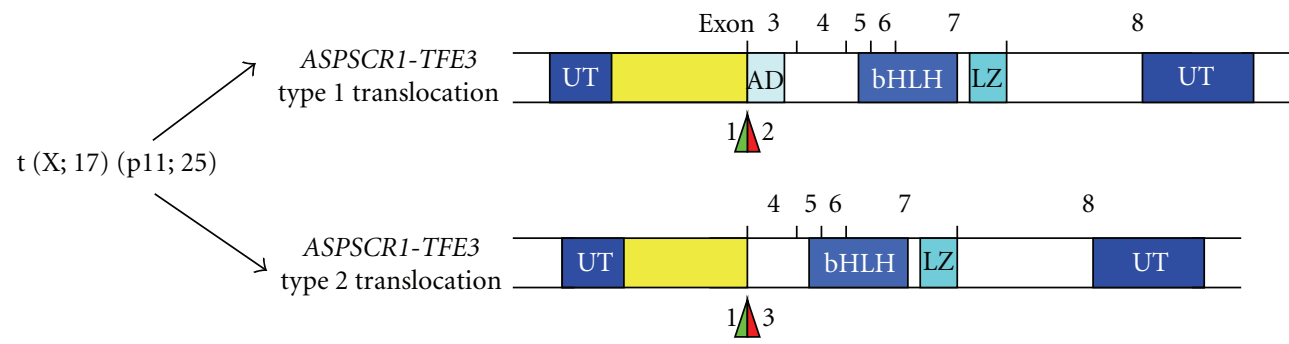

(b)

Figure 1: The $\mathrm{t}(\mathrm{X} ; 17)(\mathrm{p} 11 ; 25)$ translocation. (a) The ASPSCR-1 gene is located at chromosome 17q25 and the TFE3 gene at Xp11. The breakpoint found in the ASPSCR-1 gene is marked at "1", and the two defined breakpoints in the TFE3 gene are marked " 2 " and " 3 ". (b) Following translocation, two variants of the ASPSCR-1-TFE3 fusion gene can be created. The Type 1 translocation retains the N-terminal activation domain of the TFE3 gene.

from each of these translocations. Interestingly, $15 \%$ of cases of renal cell carcinomas in which TFE3 gene fusions are detected is associated with prior exposure to chemotherapy [30]. A strong association between prior chemotherapy and the subsequent development of ASPS has not been demonstrated.

The ASPSCR-1 gene has been alternatively termed in the literature TUG (Tether-containing UBX domain for GLUT4), ASPL (alveolar soft part sarcoma locus), UBXN9, UBXD9, and FLJ45380. This protein is expressed ubiquitously, though it has highest expression in the adult heart and skeletal muscle [22]. For a number of years following the discovery of the ASPSCR1-TFE3 translocation, the function of the ASPSCR-1 gene product was largely unknown; there are now data that show that it functions as a tether which interacts with the glucose transporter type 4 (GLUT4) and cellular/organellar membranes [31]. The ASPSCR-1 protein appears to sequester the GLUT4 in intracellular vesicles in muscle and adipocytes in the absence of insulin and facilitates redistribution of this channel to the plasma membrane following insulin stimulation. In the context of a novel fusion protein, it is unclear how the anchoring functionality of ASPSCR-1 may influence the function of TEF3.

One may speculate that the novel $\mathrm{N}$-terminus of the ASPSCR1-TFE3 fusion protein may interfere with or obviate the normal activation or dimerization functions of TEF3 to the extent that normal transcription is deranged. TEF3 may bind an alternative transcription factor, leading to aberrant transcriptional programs or simply homodimerize in the absence of an activating signal and remain constitutively active. The specific role of an N-terminal segment of the TUG protein is unclear, though hypotheses could be made that the presence of this peptide alters dimerization or activation of the TEF3 peptide component. It is important to note, however, that the TFE3 gene is associated with other tumors and a number of oncogenic translocations. The $\mathrm{t}(\mathrm{X} ; 17)(\mathrm{p} 11 ; 25)$ translocation is additionally detected in some cases of perivascular epithelioid cell neoplasms (PEComas), and as mentioned above, and also is found in papillary renal cell adenocarcinomas, more frequently in the pediatric population [32]. Within this subset of renal cell adenocarcinomas, four other TFE3 gene translocations have been described, as shown Table 1 [22, 33-39]. Additionally, novel chromosomal translocations have been identified which await definition of the involved gene loci. Thus, five discrete translocations associated with oncogenesis have been identified to date, and these translocants are thought to serve diverse functions. This suggests that perhaps the loss of the native $\mathrm{N}$-terminus of the TFE3 gene is more important in tumorigenesis than the particular composition of the ectopic genetic material added to it.

In the last few years, large strides have been made in ascertaining how the unique ASPSCR-1-TEF3 fusion protein leads to tumorigenesis. Tsuda et al. (2007) identified that the ASPL-TFE3 fusion protein induces strong overexpression of the MET receptor tyrosine kinase gene in ASPS cells [40]. This group showed that in the presence of its ligand, hepatocyte growth factor (HGF), the MET receptor tyrosine kinase underwent strong autophosphorylation, activating robust 
TABLE 1: Translocations involving the TFE3 gene.

\begin{tabular}{llll}
\hline Fusion protein & Genetic translocation & Function of gene N-terminal to TFE3 & Reference \\
\hline TFE-ASPSCR-1 & $\mathrm{t}(\mathrm{X} ; 17)(\mathrm{p} 11 ; 25)$ & Tether-containing UBX domain for GLUT4 (ASPSCR-1) & Ladanyi et al. [22, 24] \\
TFE-PRCC & $\mathrm{t}(\mathrm{X} ; 1)(\mathrm{p} 11.2 ; \mathrm{q} 21.2)$ & Papillary renal cell carcinoma translocation-associated (PRCC) gene & $\begin{array}{l}\text { Weterman et al. [33] } \\
\text { and Sidhar et al. [34] }\end{array}$ \\
TFE-PSF & $\mathrm{t}(\mathrm{X} ; 1)(\mathrm{p} 11.2 ; \mathrm{p} 34)$ & PTB-associated splicing factor $(P S F)$ & Clark et al. [35] \\
TFE-NonO & $\operatorname{inv}(\mathrm{X})(\mathrm{p} 11.2 ; \mathrm{q} 12)$ & Non-POU-domain-containing, octamer-binding (NonO) gene & Clark et al. [35] \\
TFE-CLTC & $\mathrm{t}(\mathrm{X} ; 17)(\mathrm{p} 11.2 ; \mathrm{q} 23)$ & Clathrin heavy-chain $(C L T C)$ gene & Argani et al. [36] \\
Unknown & $\mathrm{t}(\mathrm{X} ; 10)(\mathrm{p} 11.2 ; \mathrm{q} 23)$ & Unknown & Dijkhuizen et al. [37] \\
Unknown & $\mathrm{t}(\mathrm{X} ; 3)(\mathrm{p} 11 ; \mathrm{q} 23)$ & Unknown & Argani et al. [38] \\
Unknown & $\mathrm{t}(\mathrm{X} ; 19)(\mathrm{p} 11.2 ; \mathrm{q} 13.1)$ & Unknown & Armah et al. [39] \\
\hline
\end{tabular}

downstream signaling of the MAP kinase and PI3K/Akt pathways (Figure 2). Inhibiting expression of MET by RNA interference or a specific inhibitor abolished the HGF-dependent MET activation, leading to decreased cell growth. These data provide a mechanism, whereby the presence of the ASPSCR1-TFE3 fusion protein could potentially induce cell mitosis. Interestingly, the PSF-TFE3 and NonO-TFE3 fusion proteins also activated this promoter, again implicating TEF3 as the primary determinant of this phenomenon. As mentioned, TEF3 may have broad roles in regulating mitosis and the release of cell cycle blockade; additional parallel signaling circuits may be similarly activated. Nonetheless, the induction of the MET receptor tyrosine kinase pathway by the ASPSCR-1-TFE3 fusion protein represents a major advance in our understanding of this tumor.

\section{Contemporary Therapeutic Approaches}

The majority of clinical data concerning the outcomes for those diagnosed with ASPS comes from large case series spanning many decades, given the rarity of this tumor. Lieberman et al. [2] provide the largest descriptive study of patients with ASPS to date; data from 102 patients with ASPS were collected from the years 1923 to 1986, and their outcomes are studied. Aggregate 5-year survival was $62 \%$ at 5 years and $18 \%$ at 20 years (median 7 years). Of 69 patients without metastasis at diagnosis, $60 \%$ remained free of metastasis after 5 years and 15\% after 20 years (median 6 years). Those who developed metastases following diagnosis had a median survival of 2 years after discovery. The most common sites for metastasis noted were lung, bone, and brain, though it was noted that brain metastasis was never detected in the absence of lung metastasis. Of those with metastasis at diagnosis, the median survival was 3 years.

This case series provided the first evidence that surgical intervention appears to be the only clinically effective therapy for the treatment of ASPS. Indeed, adjuvant therapies including chemotherapy or radiation therapy yielded no significant difference in overall patient survival regardless of stage, nor in the context of the presence, absence, or later development of metastasis. In this study, of 91 patients with localized disease who underwent primary surgical excision, 18 patients were also treated with radiotherapy, 2 with chemotherapy, and 2 patients with both modalities. The rate of local recurrence was not significantly different compared to those treated with no adjuvant therapy. Similarly, adjuvant therapy yielded no significant difference in the number of patients who went on to develop metastases. Finally, no treatment modality, including chemotherapy, radiation therapy, or surgical resection appeared to provide a survival benefit compared to those who were not treated after the development of metastasis.

A number of similar conclusions were drawn from a more recent study. Portera et al. [1] report data from 74 patients collected over almost 40 years; in this study, $65 \%$ presented with American Joint Committee on Cancer (AJCC) stage IV (metastatic) disease and the rest with AJCC Stage II or III disease. In this series, those with nonmetastatic disease were treated with surgery alone ( 9 patients) or surgery plus external beam radiotherapy (10 patients). Three patients were also given neoadjuvant doxorubicin prior to surgical resection. For those with localized ASPS, the 5-year local recurrence free, distant recurrence free, disease free, and overall survival rates were $88 \%, 84 \%, 71 \%$, and $87 \%$, respectively. Over a decade, 2 of 22 patients with localized disease developed local recurrence, and 3 developed lung metastasis, reflecting percentages similar to those reported by Lieberman et al. From these data, radiation therapy again did not appear to greatly influence survival or the development of metastases, though low patient numbers preclude definitive conclusions.

Of 48 patients presenting with Stage IV disease in this study, $73 \%$ had metastasis to one organ, which was the lung in $\sim 90 \%$ of cases. In those with more than one site of metastasis, the lung was always involved, and brain metastases were found in 9 of 29 patients. Twenty-six patients of 33 with Stage IV disease were given systemic chemotherapy which included vincristine and/or cyclophosphamide (prior to 1970) or doxorubicin-based therapy. The majority of patients treated with chemotherapy (58\%) developed disease progression. This population median survival was 40 months, with a 5year survival rate of $20 \%$. These data again mirror previously described data.

Importantly, this case series showed that with more "modern" chemotherapy regimens utilizing vincristine, cyclophosphamide, or doxorubicin, clinical response was disappointing. Among the 26 patients with Stage IV disease who received chemotherapy and the 3 patients with localized 


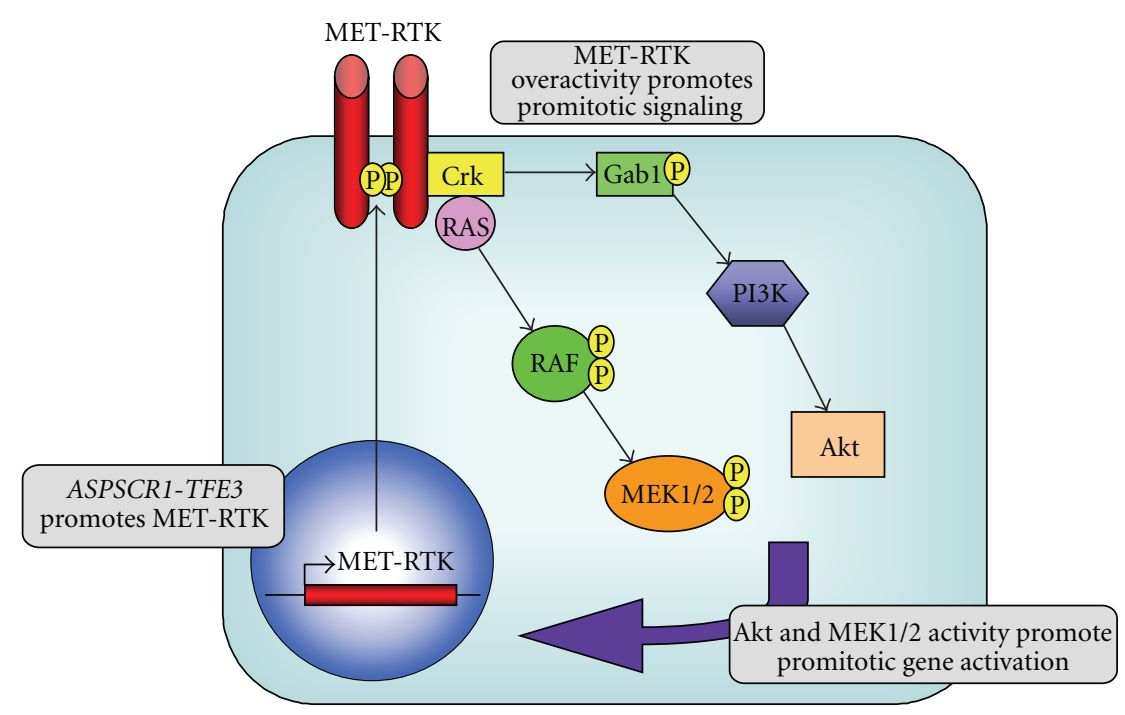

(a)

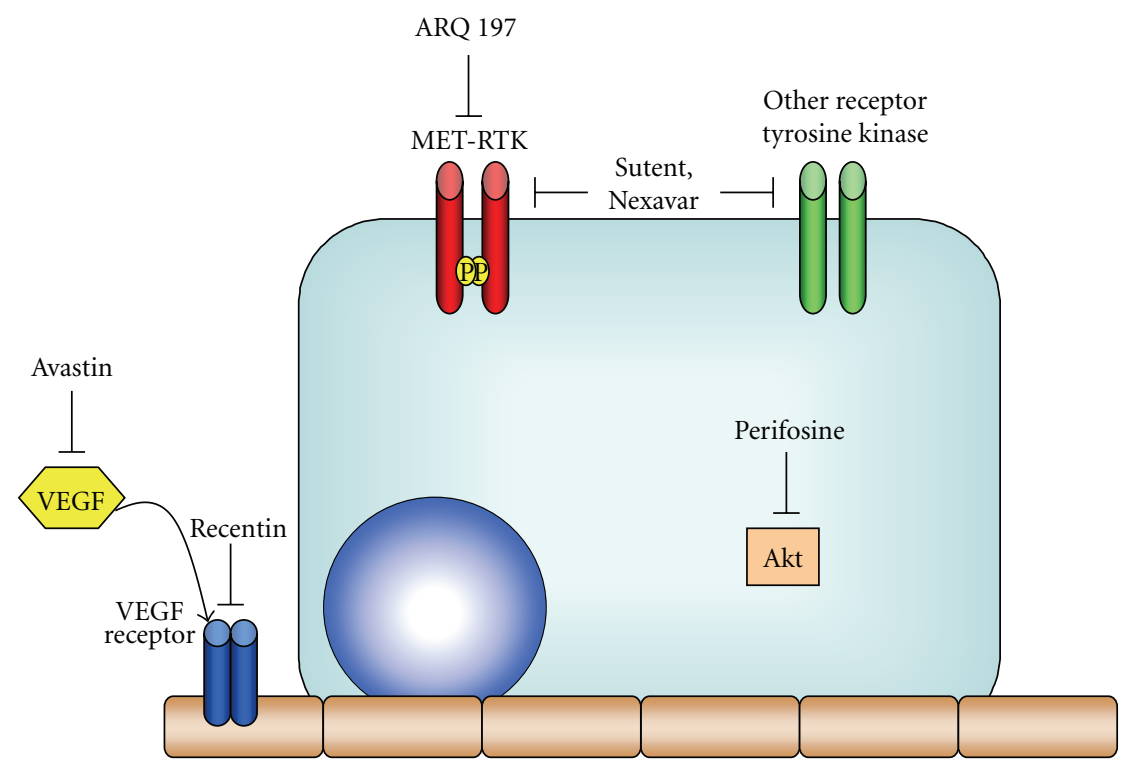

(b)

FIGURE 2: Signaling schematic for ASPS tumors and novel therapeutic targets. (a) Overexpression of the MET tyrosine receptor kinase is induced by the ASPSCR1-TFE3 fusion protein. This leads to intracellular activation of the promitotic growth kinases Akt and MEK1/2; these in turn lead to unchecked cellular proliferation. (b) Targets for novel therapeutics include the VEGF tyrosine kinase receptor and its ligand (Recentin and Avastin, resp.), as well as tumor cell receptor tyrosine kinases (Sutent, Nexavar, and ARQ 197). Perifosine is a unique inhibitor of the Akt kinase.

disease who received neoadjuvant doxorubicin-based systemic therapy prior to resection, only a single patient stage IV responded, though there was a complete response. Chemotherapy yielded no minor or incomplete responses. Thus, this study provided little evidence that contemporary systemic chemotherapy elicits a survival benefit.

Finally, Kayton et al. [6] describe data from 20 patients collected over 30 years. These patients ranged in age from 6 to 25 , with $35 \%$ of patients presenting with Intergroup Rhabdomyosarcoma Study stage IV (metastatic) disease. Patients with IRS Stage I (local) disease underwent surgery alone, and none had evidence of local recurrence at followup, which ranged from 4 to 290 months; $20 \%$ of patients, however, developed detectable metastases at followup. For those with IRS Stage IV disease, a variety of approaches including radiation therapy to the primary tumor or metastases, chemotherapy, and excision of the primary mass \pm metastasectomy were attempted. The authors note no partial or complete responses to a wide variety of chemotherapy regimens attempted, including antimetabolites, alkylating agents, mitotic inhibitors, anthracyclines, or biologic agents. Similarly, small numbers precluded clear conclusions regarding radiation therapy. The aggregate 5-year survival for all patients with ASPS was 83\%, with 5-year progression-free survival 
of $22 \%$; of note, those presenting with a primary site tumor $>5 \mathrm{~cm}$ in size all died before 5-year followup, whereas those with tumor size $<5 \mathrm{~cm}$ had a $\sim 70 \%$ progression-free survival. The data from this case series emphasize the importance of complete microscopic resection with negative margins in those with early stage disease, as well as the large bearing tumor burden plays in determining survival. Furthermore, for patients who present with metastases, the authors note very short progression-free survival (median 12.5 months) but much longer time of overall followup (median 36 months). This may reflect the indolent nature of the disease, rather than the effectiveness of surgical resection per se.

From these reports, there are no data to support the use of any modality of therapy aside from surgery for ASPS; no significant survival advantages have been achieved by utilizing chemotherapy or radiation for patients who have local or metastatic disease at the time of diagnosis as compared to patients who are not treated. The authors of these studies, however, note the importance of palliation, when necessary, and that though there are no data demonstrating survival benefit, radiation therapy should be considered in specific cases where there is a large primary tumor burden. Our recommendations based on these data are expectant observation when metastatic disease is low volume and nonprogressive on serial imaging.

\section{Targeted Therapies and Clinical Trials}

The resistance of ASPS tumors to conventional chemotherapies and radiation makes this type of tumor challenging to treat; however, a number of exciting clinical trials are underway which are investigating novel targeted therapies. These newer agents offer many advantages over traditional chemotherapeutic agents and radiotherapy, such as reduced toxicity and daily outpatient use. The theoretical ability to indefinitely continue minimally toxic therapy becomes especially important, given the indolent nature of ASPS even in the context of metastatic disease. A number of trials underway currently seek to focus on the overactivity of the MET receptor tyrosine kinase gene induced by the ASPSCR1TFE3 fusion protein. In addition, the vascular nature of this tumor also suggests a potential role for antiangiogenic agents.

One such trial focused on ARQ-197 (ArQule), a selective inhibitor of the c-Met receptor tyrosine kinase. This drug was tested in a Phase II study (NCI Clinical Trial NCT00557609) examining the drug's effect on Microphthalmia Transcription Factor Family (MiT) tumors, which include ASPS, clear cell carcinoma, and renal cell carcinomas bearing a TFE3 translocation [41]. Preliminary data presented at the 2009 American Society of Clinical Oncology indicated that 15 of 17 patients treated with ARQ-197 demonstrated stable disease at $\sim 29$ weeks of therapy, with a disease control rate of $\sim 80 \%$ [42]. This drug has an excellent safety trial following the completion of three Phase I trials, and although data are not mature, they appear promising.

Along these same lines, multitargeted tyrosine kinase inhibitors have also been investigated in small trials; sunitinib malate (SU11248, Sutent, Pfizer), a multitargeted RTK inhibitor with antiangiogenic properties approved for treatment of GIST and renal cancer, was recently trialed in 8 patients [43]. Five patients showed partial response, one had stable disease and one progressed. Similarly, a phase II trial with sorafenib (Nexavar, Bayer, NCI Trial NCT00330421), another multitargeted tyrosine kinase inhibitor, showed that in 28 patients, 12 had partial response and 6 had stable disease, for a disease control rate of 78\% [44]. Other clinical trials underway for the treatment of ASPS include the Akt inhibitor, KRX-0401 (Perifosine, Keryx/AOI, NCI Clinical Trial NCT00401388), and antiangiogenic approaches such as bevacizumab (Avastin, Genentech) or Cediranib (Recentin, AstraZeneca) [45-47]. Preliminary data from two phase II trials using Cediranib (Study code 2171IL0038) also presented at the 2009 American Society of Clinical Oncology showed that four of seven patients had a partial response, two patients had a confirmed reduction in tumor size, and one patient demonstrated stable disease [48]. Further clinical trials for Cediranib are currently open to accrual (NCI Clinical Trials NCT01337401 and NCT00942877) $[49,50]$.

\section{Future Directions}

The discovery of further novel therapeutic targets has been aided by a number of recent reports providing broad and targeted gene expression and immunohistochemical arrays. Together, Stockwin et al. and Lazar et al. provide comprehensive studies characterizing gene expression profiles of ASPS, with a focus on a number of key players involved in angiogenesis, cell proliferation, and metastasis [51, 52]. For instance, these manuscripts both identified upregulation of MDK (midkine or neurite growth-promoting factor-2) and Jag-1 (Jagged-1), which are regulators of angiogenesis. MDK is a low molecular weight growth factor which antagonizes VEGF signaling and that appears to be upregulated in multiple solid tumors; Jag-1, in contrast, is the ligand for the Notch-1 receptor and is a potent proangiogenic signal [53-56]. There are numerous suggestions that both these molecules represent excellent targets for novel therapeutics, and there are preclinical data that are promising [57-59]. Stockwin et al. also demonstrated the expression of GPNMB, a transmembrane protein which bears homology to the melanoma antigen pMEL17. CDX-011, or Glembatumumab (Celldex), is an antibody targeted against GPNMB (Transmembrane Protein NMB); this antibody was conjugated to vedotin (monomethyl auristatin E), a highly potent antimitotic agent, in recent Phase 2 trials for advanced breast cancer and late-stage melanoma $[60,61]$. These data provide a rational basis for use of this drug in ASPS. Finally, Martignoni et al. demonstrate that all tested samples of alveolar soft part sarcomas diffusely express cathepsin $\mathrm{K}$, whose expression is driven by MITF in osteoclasts; interestingly, renal cell carcinomas with the same ASPSCR1TFE3 translocation do not detectably express this protease [62]. Argani et al. also reported the expression of cathepsin $\mathrm{K}$ in PEComas [32]. Odanacatib (MK-0822, Merck) is a monoclonal antibody against cathepsin $\mathrm{K}$ and has been studied in women with breast cancer with bony metastases; 
again, cathepsin $\mathrm{K}$ may represent a potential therapeutic target [63].

\section{Conclusions}

In summary, alveolar soft part sarcomas are rare, unique malignancies which grow indolently and remain difficult to treat despite decades of clinical experience. Recent data have linked the specific $t(X ; 17)(p 11 ; 25)$ translocation found in all ASPS tumors studied to the overexpression of the promitotic MET receptor tyrosine kinase, providing a model for tumorigenesis. At this point, surgical methods are the most efficacious means of disease treatment; there are no convincing data in support of conventional chemotherapy or radiation therapy. New molecular therapies targeted to tyrosine receptor kinases and antiangiogenic agents have yielded promising data thus far, and these next-generation therapies may soon comprise first-line treatment for this tumor type.

\section{Conflict of Interests}

The authors of this paper declare no financial conflict of interests.

\section{References}

[1] C. A. Portera, V. Ho, S. R. Patel et al., "Alveolar soft part sarcoma: clinical course and patterns of metastasis in 70 patients treated at a single institution," Cancer, vol. 91, no. 3, pp. 585$591,2001$.

[2] P. H. Lieberman, M. F. Brennan, M. Kimmel, R. A. Erlandson, P. Garin-Chesa, and B. Y. Flehinger, "Alveolar soft-part sarcoma. A clinico-pathologic study of half a century," Cancer, vol. 63, no. 1, pp. 1-13, 1989.

[3] N. G. Ordóñez and B. Mackay, "Alveolar soft-part sarcoma: a review of the pathology and histogenesis," Ultrastructural Pathology, vol. 22, no. 4, pp. 275-292, 1998.

[4] N. G. Ordóñez, "Alveolar soft part sarcoma: a review and update," Advances in Anatomic Pathology, vol. 6, no. 3, pp. 125$139,1999$.

[5] M. Casanova, A. Ferrari, G. Bisogno et al., "Alveolar soft part sarcoma in children and adolescents: a report from the softtissue sarcoma italian cooperative group," Annals of Oncology, vol. 11, no. 11, pp. 1445-1449, 2000.

[6] M. L. Kayton, P. Meyers, L. H. Wexler, W. L. Gerald, and M. P. LaQuaglia, "Clinical presentation, treatment, and outcome of alveolar soft part sarcoma in children, adolescents, and young adults," Journal of Pediatric Surgery, vol. 41, no. 1, pp. 187-193, 2006.

[7] A. Ogose, T. Morita, T. Hotta et al., "Brain metastases in musculoskeletal sarcomas," Japanese Journal of Clinical Oncology, vol. 29, no. 5, pp. 245-247, 1999.

[8] M. Salvati, L. Cervoni, R. Caruso, F. M. Gagliardi, and R. Delfini, "Sarcoma metastatic to the brain: a series of 15 cases," Surgical Neurology, vol. 49, no. 4, pp. 441-444, 1998.

[9] H. L. Evans, "Alveolar soft-part sarcoma. A study of 13 typical examples and one with a histologically atypical component," Cancer, vol. 55, no. 4, pp. 912-917, 1985.

[10] J. G. Lorigan, F. N. O'Keeffe, H. L. Evans, and S. Wallace, "The radiologic manifestations of alveolar soft-part sarcoma,"
American Journal of Roentgenology, vol. 153, no. 2, pp. 335339, 1989.

[11] W. M. Christopherson, F. W. Foote, and F. W. Stewart, "Alveolar soft-part sarcomas; structurally characteristic tumors of uncertain histogenesis," Cancer, vol. 5, no. 1, pp. 100-111, 1952.

[12] G. Tallini, D. M. Parham, P. Dias, C. Cordon-Cardo, P. J. Houghton, and J. Rosai, "Myogenic regulatory protein expression in adult soft tissue sarcomas: a sensitive and specific marker of skeletal muscle differentiation," American Journal of Pathology, vol. 144, no. 4, pp. 693-701, 1994.

[13] N. P. Wang, C. E. Bacchi, J. J. Jiang, M. A. McNutt, and A. M. Gown, "Does alveolar soft-part sarcoma exhibit skeletal muscle differentiation? An immunocytochemical and biochemical study of myogenic regulatory protein expression," Modern Pathology, vol. 9, no. 5, pp. 496-506, 1996.

[14] J. Rosai, P. Dias, D. M. Parham, D. N. Shapiro, and P. Houghton, "MyoD1 protein expression in alveolar soft part sarcoma as confirmatory evidence of its skeletal muscle nature," American Journal of Surgical Pathology, vol. 15, no. 10, pp. 974-981, 1991.

[15] J. A. Gómez, M. B. Amin, J. Y. Ro, M. D. Linden, M. W. Lee, and R. J. Zarbo, "Immunohistochemical profile of Myogenin and MyoD1 does not support skeletal muscle lineage in alveolar soft part sarcoma: a study of 19 tumors," Archives of Pathology and Laboratory Medicine, vol. 123, no. 6, pp. 503507, 1999.

[16] G. P. Nielsen, E. Oliva, R. H. Young, A. E. Rosenberg, G. R. Dickersin, and R. E. Scully, "Alveolar soft-part sarcoma of the female genital tract: a report of nine cases and review of the literature," International Journal of Gynecological Pathology, vol. 14, no. 4, pp. 283-292, 1995.

[17] S. Yagihashi, N. Yagihashi, Y. Hase, K. Nagai, and A. AlguacilGarcia, "Primary alveolar soft-part sarcoma of stomach," American Journal of Surgical Pathology, vol. 15, no. 4, pp. 399406, 1991.

[18] J. Wu, D. A. Brinker, M. Haas, E. A. Montgomery, and P. Argani, "Primary alveolar soft part sarcoma (ASPS) of the breast: report of a deceptive case with xanthomatous features confirmed by TFE3 immunohistochemistry and electron microscopy," International Journal of Surgical Pathology, vol. 13, no. 1, pp. 81-85, 2005.

[19] J. C. Fanburg-Smith, M. Miettinen, A. L. Folpe, S. W. Weiss, and E. L. B. Childers, "Lingual alveolar soft part sarcoma; 14 cases: novel clinical and morphological observations," Histopathology, vol. 45, no. 5, pp. 526-537, 2004.

[20] H. Smetana and W. Scott, "Malignant tumors of nonchromaffin paraganglia," Military Surgery, vol. 109, no. 4, pp. 330-349, 1951.

[21] M. Ladanyi, C. R. Antonescu, M. Drobnjak et al., "The precrystalline cytoplasmic granules of alveolar soft part sarcoma contain monocarboxylate transporter 1 and CD147," American Journal of Pathology, vol. 160, no. 4, pp. 1215-1221, 2002.

[22] M. Ladanyi, M. Y. Lui, C. R. Antonescu et al., "The $\operatorname{der}(17) t(X ; 17)(\mathrm{p} 11 ; \mathrm{q} 25)$ of human alveolar soft part sarcoma fuses the TFE3 transcription factor gene to ASPL, a novel gene at 17q25," Oncogene, vol. 20, no. 1, pp. 48-57, 2001.

[23] H. Y. Huang, M. Y. Lui, and M. Ladanyi, "Nonrandom cellcycle timing of a somatic chromosomal translocation: the $\mathrm{t}(\mathrm{X} ; 17)$ of alveolar soft-part sarcoma occurs in G2," Genes Chromosomes and Cancer, vol. 44, no. 2, pp. 170-176, 2005.

[24] P. Argani, C. R. Antonescu, P. B. Illei et al., "Primary renal neoplasms with the ASPL-TFE3 gene fusion of alveolar soft part sarcoma: a distinctive tumor entity previously included 
among renal cell carcinomas of children and adolescents," American Journal of Pathology, vol. 159, no. 1, pp. 179-192, 2001.

[25] T. J. Hemesath, E. Steingrimsson, G. McGill et al., "Microphthalmia, a critical factor in melanocyte development, defines a discrete transcription factor family," Genes and Development, vol. 8, no. 22, pp. 2770-2780, 1994.

[26] R. P. Kuiper, M. Schepens, J. Thijssen, E. F. P. M. Schoenmakers, and A. G. van Kessel, "Regulation of the MiTF/TFE bHLHLZ transcription factors through restricted spatial expression and alternative splicing of functional domains," Nucleic Acids Research, vol. 32, no. 8, pp. 2315-2322, 2004.

[27] S. M. Nijman, E. M. Hijmans, S. El Messaoudi, M. M. W. Van Dongen, C. Sardet, and R. Bernards, "A functional genetic screen identifies TFE3 as a gene that confers resistance to the anti-proliferative effects of the retinoblastoma protein and transforming growth factor- $\beta$," Journal of Biological Chemistry, vol. 281, no. 31, pp. 21582-21587, 2006.

[28] H. Beckmann, L. K. Su, and T. Kadesch, “TFE3: a helix-loophelix protein that activates transcription through the immunoglobulin enhancer uE3 motif," Genes and Development, vol. 4, no. 2, pp. 167-179, 1990.

[29] C. Roman, L. Cohn, and K. Calame, "A dominant negative form of transcription activator mTFE3 created by differential splicing," Science, vol. 254, no. 5028, pp. 94-97, 1991.

[30] P. Argani, M. Laé, E. T. Ballard et al., "Translocation carcinomas of the kidney after chemotherapy in childhood," Journal of Clinical Oncology, vol. 24, no. 10, pp. 1529-1534, 2006.

[31] G. Alexandru, J. Graumann, G. T. Smith, N. J. Kolawa, R. Fang, and R. J. Deshaies, "UBXD7 binds multiple ubiquitin ligases and implicates p97 in HIF1 $\alpha$ turnover," Cell, vol. 134, no. 5, pp. 804-816, 2008.

[32] P. Argani, S. Aulmann, P. B. Illei et al., "A distinctive subset of PEComas harbors TFE3 gene fusions," American Journal of Surgical Pathology, vol. 34, no. 10, pp. 1395-1406, 2010.

[33] M. A. J. Weterman, M. Wilbrink, and A. G. Van Kessel, "Fusion of the transcription factor TFE3 gene to a novel gene, PRCC, in $\mathrm{t}(\mathrm{X} ; 1)(\mathrm{p} 11 ; \mathrm{q} 21)$-positive papillary renal cell carcinomas," Proceedings of the National Academy of Sciences of the United States of America, vol. 93, no. 26, pp. 15294-15298, 1996.

[34] S. K. Sidhar, J. Clark, S. Gill et al., "The $\mathrm{t}(\mathrm{X} ; 1)(\mathrm{p} 11.2 ; \mathrm{q} 21.2)$ translocation in papillary renal sell carcinoma fuses a novel gene PRCC to the TFE3 transcription factor gene," Human Molecular Genetics, vol. 5, no. 9, pp. 1333-1338, 1996.

[35] J. Clark, Y. J. Lu, S. K. Sidhar et al., "Fusion of splicing factor genes PSF and NonO ( $\left.\mathrm{p} 54^{n r b}\right)$ to the TFE3 gene in papillary renal cell carcinoma," Oncogene, vol. 15, no. 18, pp. 22332239, 1997.

[36] P. Argani, M. Y. Lui, J. Couturier, R. Bouvier, J. C. Fournet, and M. Ladanyi, "A novel CLTC-TFE3 gene fusion in pediatric renal adenocarcinoma with $\mathrm{t}(\mathrm{X} ; 17)(\mathrm{p} 11.2 ; \mathrm{q} 23)$, Oncogene, vol. 22, no. 34, pp. 5374-5378, 2003.

[37] T. Dijkhuizen, E. van den Berg, M. Wilbrink et al., "Distinct Xp11.2 breakpoints in two renal cell carcinomas exhibiting $\mathrm{X}$; autosome translocations," Genes Chromosomes and Cancer, vol. 14, no. 1, pp. 43-50, 1995.

[38] P. Argani, S. Olgac, S. K. Tickoo et al., "Xp11 Translocation renal cell carcinoma in adults: expanded clinical, pathologic, and genetic spectrum," American Journal of Surgical Pathology, vol. 31, no. 8, pp. 1149-1160, 2007.

[39] H. B. Armah, A. V. Parwani, U. Surti, and S. I. Bastacky, "Xp11.2 translocation renal cell carcinoma occurring during pregnancy with a novel translocation involving chromosome
19: a case report with review of the literature," Diagnostic $\mathrm{Pa}$ thology, vol. 4, no. 1, article 15, 2009.

[40] M. Tsuda, I. J. Davis, P. Argani et al., "TFE3 fusions activate MET signaling by transcriptional up-regulation, defining another class of tumors as candidates for therapeutic MET inhibition," Cancer Research, vol. 67, no. 3, pp. 919-929, 2007.

[41] "A phase 2 study of ARQ 197 in patients with microphthalmia transcription factor associated tumors," http://www.clinicaltrials.gov/show/NCT00557609.

[42] J. Goldberg, G. D. Demetri, E. Choy et al., "Preliminary results from a phase II study of ARQ 197 in patients with microphthalmia transcription factor family (MiT)-associated tumors," Journal of Clinical Oncology, vol. 27, no. 15s, 2009, (suppl; abstr 10502).

[43] E. Palassini, S. Stacchiotti, T. Negri et al., "Sunitinib malate (SM) in alveolar soft part sarcoma (ASPS)," Journal of Clinical Oncology, vol. 28, no. 15s, 2010, (suppl; abstr 10014).

[44] "Sorafenib in Treating Patients With Soft Tissue Sarcomas," http://www.clinicaltrials.gov/ct/show/NCT00330421.

[45] "A Trial of Perifosine in Patients With Chemo-Insensitive Sarcomas," http://www.clinicaltrials.gov/ct2/show/NCT00401388.

[46] A. A. Azizi, C. Haberler, T. Czech et al., "Vascular-endothelialgrowth-factor (VEGF) expression and possible response to angiogenesis inhibitor bevacizumab in metastatic alveolar soft part sarcoma," Lancet Oncology, vol. 7, no. 6, pp. 521-523, 2006.

[47] D. T. Vistica, M. Hollingshead, S. D. Borgel et al., "Therapeutic vulnerability of an in vivo model of alveolar soft part sarcoma (ASPS) to antiangiogenic therapy," Journal of Pediatric Hematology/Oncology, vol. 31, no. 8, pp. 561-570, 2009.

[48] K. Gardner, I. Judson, M. Leahy et al., "Activity of cediranib, a highly potent and selective VEGF signaling inhibitor, in alveolar soft part sarcoma," Journal of Clinical Oncology, vol. 27, no. 15s, 2009, (suppl; abstr 10523).

[49] "A Trial of Cediranib in the Treatment of Patients With Alveolar Soft Part Sarcoma (CASPS)," http://www.clinicaltrials.gov/ ct2/show/NCT01337401.

[50] "Phase II Study of Cediranib (AZD2171) in Patients With Alveolar Soft Part Sarcoma," http://www.clinicaltrials.gov/ct2/ show/NCT00942877.

[51] L. H. Stockwin, D. T. Vistica, S. Kenney et al., "Gene expression profiling of alveolar soft-part sarcoma (ASPS)," BMC Cancer, vol. 9, p. 22, 2009.

[52] A. J. Lazar, P. Das, D. Tuvin et al., "Angiogenesis-promoting gene patterns in alveolar soft part sarcoma," Clinical Cancer Research, vol. 13, no. 24, pp. 7314-7321, 2007.

[53] J. Kerzerho, O. Adotevi, F. A. Castelli et al., "The angiogenic growth factor and biomarker midkine is a tumor-shared antigen," Journal of Immunology, vol. 185, no. 1, pp. 418-423, 2010.

[54] E. H. van der Horst, B. T. Frank, L. Chinn et al., "The growth factor midkine antagonizes VEGF signaling in vitro and in vivo," Neoplasia, vol. 10, no. 4, pp. 340-346, 2008.

[55] R. Benedito, C. Roca, I. Sörensen et al., "The notch ligands Dll4 and jagged 1 have opposing effects on angiogenesis," Cell, vol. 137, no. 6, pp. 1124-1135, 2009.

[56] J. Dufraine, Y. Funahashi, and J. Kitajewski, "Notch signaling regulates tumor angiogenesis by diverse mechanisms," Oncogene, vol. 27, no. 38, pp. 5132-5137, 2008.

[57] A. D. Steg, A. A. Katre, B. Goodman et al., "Targeting the Notch ligand jagged1 in both tumor cells and stroma in ovarian cancer," Clinical Cancer Research, vol. 17, no. 17, pp. 5674-5685, 2011. 
[58] A. R. Maldonado, C. Klanke, A. G. Jegga et al., "Molecular engineering and validation of an oncolytic herpes simplex virus type 1 transcriptionally targeted to midkine-positive tumors," Journal of Gene Medicine, vol. 12, no. 7, pp. 613-623, 2010.

[59] Y. Adachi, P. N. Reynolds, M. Yamamoto et al., "Midkine promoter-based adenoviral vector gene delivery for pediatric solid tumors," Cancer Research, vol. 60, no. 16, pp. 4305-4310, 2000.

[60] "Study of CR011-vcMMAE to Treat Locally Advanced or Metastatic Breast Cancer," http://www.clinicaltrials.gov/ct2/ show/NCT00704158.

[61] "A Phase I/II Study of CR011-vcMMAE in Subjects With Unresectable Stage III or Stage IV Melanoma," http://www.clinicaltrials.gov/ct2/show/NCT00412828.

[62] G. Martignoni, S. Gobbo, P. Camparo et al., "Differential expression of cathpsin $\mathrm{K}$ in neoplasms harboring TFE3 gene fusions," Modern Pathology, vol. 24, no. 10, pp. 1313-1319, 2011.

[63] "Study to Examine the Effects of an Experimental Drug on Women With Breast Cancer and Metastatic Bone Disease (MBD)," http://www.clinicaltrials.gov/ct/show/NCT00399802. 


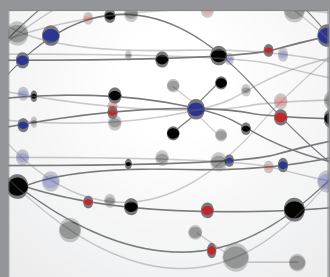

The Scientific World Journal
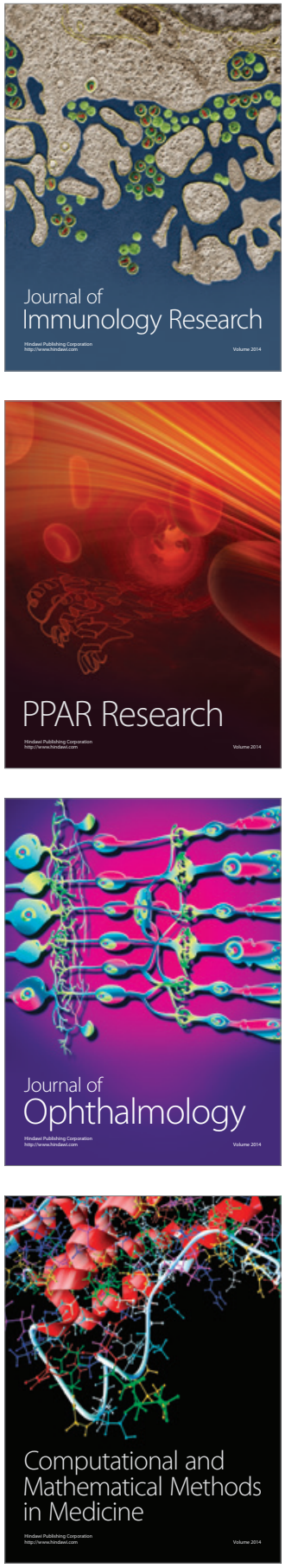

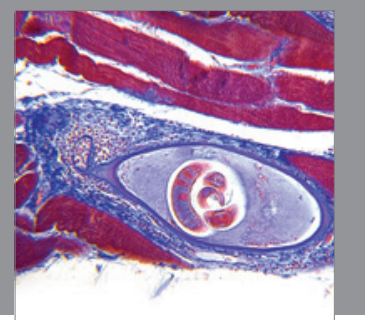

Gastroenterology

Research and Practice
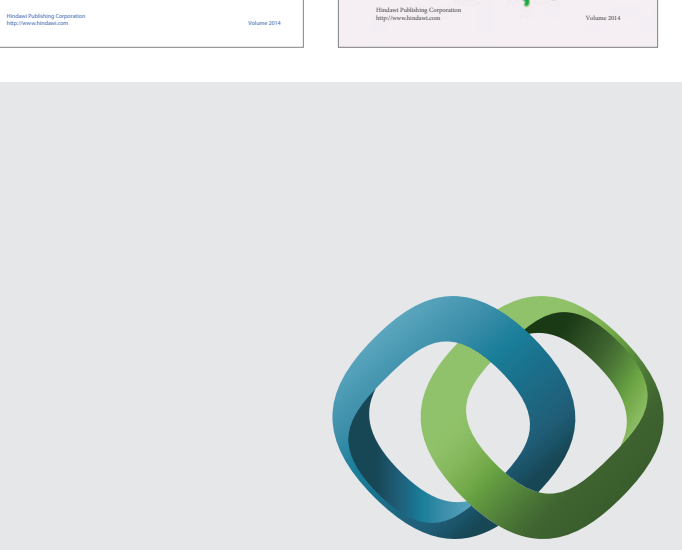

\section{Hindawi}

Submit your manuscripts at

http://www.hindawi.com
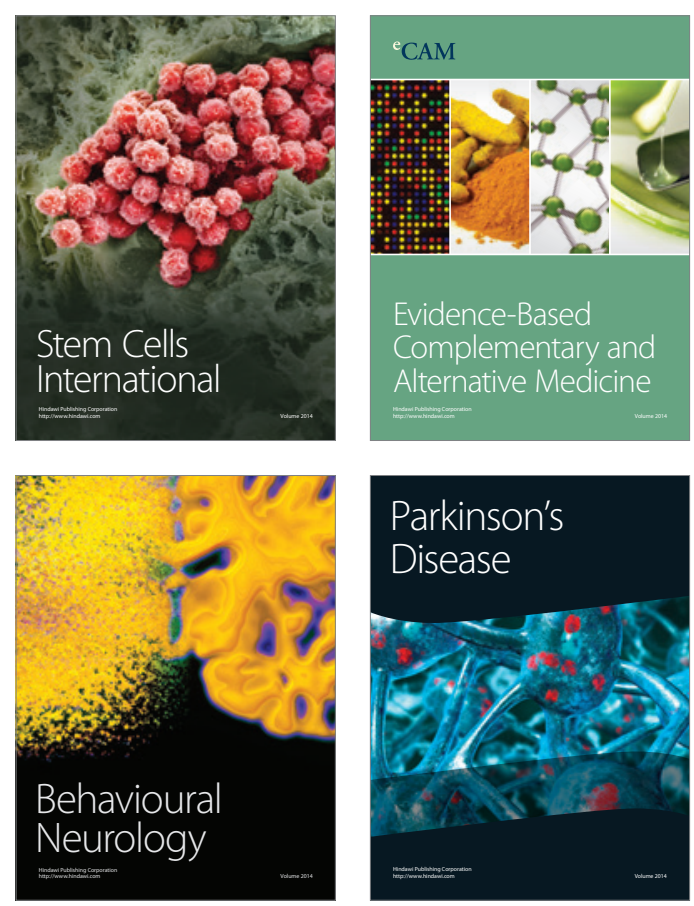

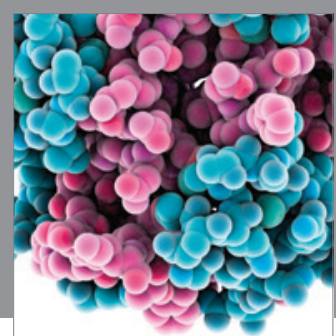

Journal of
Diabetes Research

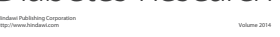

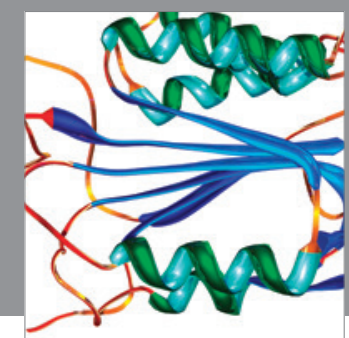

Disease Markers
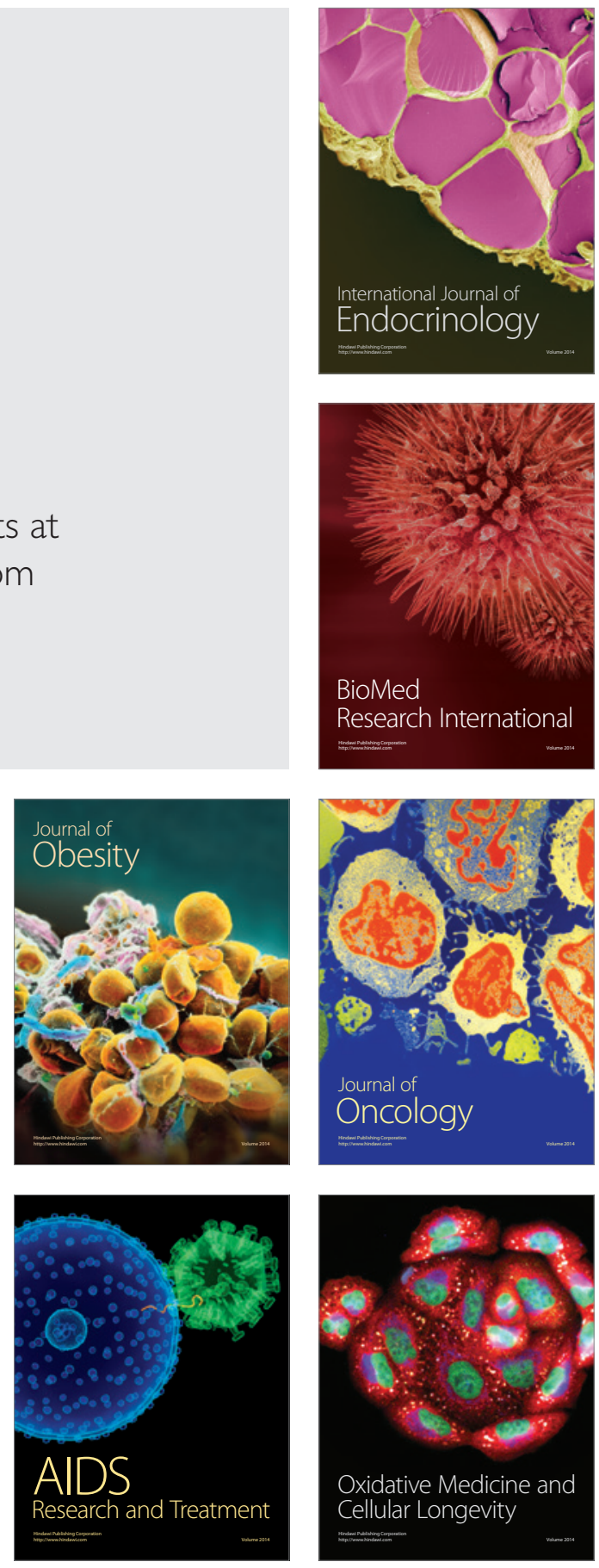